EPJ Web of Conferences 70, 00025 (2014)

DOI: $10.1051 /$ epjconf/ 20147000025

(C) Owned by the authors, published by EDP Sciences, 2014

\title{
Limits on compact halo objects as dark matter from gravitational microlensing
}

\author{
Philippe Jetzer ${ }^{1}$ \\ ${ }^{1}$ Institute of Theoretical Physics \\ University of Zürich, Winterthurerstarsse 190, CH-8057 Zürich, Switzerland \\ E-mail: jetzer@physik.uzh.ch
}

\begin{abstract}
Microlensing started with the seminal paper by Paczyński in 1986 [1], first with observations towards the Large Magellanic Cloud and the galactic bulge. Since then many other targets have been observed and new applications have been found. In particular, it turned out to be a powerful method to detect planets in our galaxy and even in the nearby M31. Here, we will present some results obtained so far by microlensing without being, however, exhaustive.
\end{abstract}

\section{Introduction}

Since Paczyński's original proposal [1] gravitational microlensing has been proven to be a powerful tool for the detection of the dark matter component in galactic haloes in form of MACHOs and for determining the structure of the Galaxy itself. Microlensing allows the detection of MACHOs (Massive Astrophysical Compact Halo Objects) located in the Galactic halo in the mass range $10^{-7}<M / M_{\odot}<1[2,3]$, as well as MACHOs in the disk or bulge of our Galaxy [4, 5]. In September 1993, the French collaboration EROS (Expérience de Recherche d'Objets Sombres) [6] announced the discovery of two microlensing candidates, and the American-Australian collaboration MACHO (for the collaboration they use the same acronym as for the compact objects) of one candidate [7] by monitoring several millions of stars in the Large Magellanic Cloud (LMC). The OGLE collaboration found one event towards the galactic bulge [8]. Since these first discoveries substantial progress has been done: many more observations have been carried out towards the LMC and the Small Magellanic Clouds (SMC), the galactic bulge, the Andromeda galaxy (M31), some globular clusters. Microlesing has develop into an important tool for the detection of extrasolar planets in our Galaxy and even in M31. For an update on this topic we refer, e.g., to the review by Dominik [9]. It is also a useful tool to study stellar atmospheres [10]. In the following we will present some of the results obtained so far towards different targets. This is, however, not an exhaustive review (see e.g. [11]), rather a personal choice of topics related to our own work in this field.

\section{Microlensing towards the Large Magellanic Cloud}

Since the first detection the MACHO team reported the observation of 13 to 17 events by analyzing their $\sim 6$ years of LMC data, leading to an optical depth of $\tau=1.0_{-0.3}^{+0.3} \times 10^{-7}$ [12]. Accordingly, this

This is an Open Access article distributed under the terms of the Creative Commons Attribution License 2.0, which permits unrestricted use, distribution, and reproduction in any medium, provided the original work is properly cited. 
would imply a most probable Galactic halo mass fraction of $\sim 20 \%$ in the form of MACHOs with a most likely mass in the range $0.15-0.9 M_{\odot}$ depending on the halo model. The MACHO value, however, is still substantially higher then the upper limit of $\tau<0.36 \times 10^{-7}$ presented by the EROS collaboration [13], based on the analysis of 33 million stars towards the LMC followed during $\sim 7$ years. Recently, also the OGLE collaboration has published its results based on almost 8 years of observations and finds $\tau=(0.16 \pm 0.12) \times 10^{-7}$, concluding that at most $7 \%$ of the galactic halo mass could be due to MACHOs. However, most probably the events they found are due to self-lensing or lenses located in the disk of our own Galaxy, in which case the upper limit gets even more stringent, ruling thus the MACHOs almost, if not completely out [14], leaving it rather difficult to explain the results of the MACHO collaboration [15]. Hopefully further observations will help clarifying this issue. The OGLE collaboration published recently its observations towards the SMC, where they found 3 good candidates yielding an optical depth of $\tau=(1.30 \pm 1.01) \times 10^{-7}$, which could be consistent with self-lensing in the SMC and a contribution from our own Galactic disc [16]. However, a more detailed analysis is needed.

\subsection{LMC self-lensing event rate}

The hypothesis for a self-lensing component was discussed by several authors [17-20]. The analysis of Jetzer et al. [21] and Mancini et al. [22] has shown that probably the observed microlensing events, in particular the ones by the MACHO collaboration, are distributed among different galactic components (disk, spheroid, galactic halo, LMC halo and self-lensing). This means that the lenses do not belong all to the same population and their astrophysical features can differ deeply one another.

Some of the events found by the MACHO team are most probably due to self-lensing: the event MACHO-LMC-9 is a double lens with caustic crossing [23] and its proper motion is very low, thus favouring an interpretation as a double lens within the LMC. The source star for the event MACHOLMC-14 is double [24] and this has allowed to conclude that the lens is most probably in the LMC. The expected LMC self-lensing optical depth due to these two events has been estimated to lie within the range [24] $1.1-1.8 \times 10^{-8}$, which is still below the expected optical depth for self-lensing even when considering models giving low values it. The event LMC-5 is due to a disk lens [25] and indeed the lens has even been observed with the HST. The other stars which have been microlensed were also observed but no lens could be detected, thus implying that the lens cannot be a disk star but has to be either a true halo object or a faint star or brown dwarf in the LMC itself.

We have calculated the self-lensing distributions of the rate of microlensing events with respect to the Einstein time $T_{\mathrm{E}}$, along the lines of sight towards 14 events found by the MACHO collaboration. With these distributions we have calculated the median $T_{\mathrm{E}, 50 \%}$ values of the Einstein time. In Fig. 1 we report on the $y$-axis the observed values of $T_{\mathrm{E}}$ (empty boxes) as well as the expected values for self-lensing of the median $T_{\mathrm{E}, 50 \%}$ (filled stars) evaluated along the directions of the events. On the $x$-axis we report the value of the self-lensing optical depth $\tau_{\mathrm{SL}}$ calculated towards the event position; the optical depth is growing going from the outer regions towards the center of LMC. An interesting feature emerging clearly is the decreasing trend of the expected values of the median $T_{\mathrm{E}, 50 \%}$, going from the outside fields with low values of $\tau_{\mathrm{SL}}$ towards the central fields with higher values of $\tau_{\mathrm{SL}}$. The variation of the stellar number density and the flaring of the LMC disk certainly contributes to explain this behaviour.

Based on these results we tentatively identify two subsets of events: the nine falling outside the contour line $\tau_{\mathrm{SL}}=2 \times 10^{-8}$ and the five falling inside. We note that, at glance, the two clusters have a clear-cut different collective behaviour: the measured Einstein times of the first 9 points fluctuate around a median value of 48 days, very far from the expected values of the median $T_{\mathrm{E}}$, ranging from 66 days to 78 days, with an average value of 72 days. On the contrary, for the last 5 points, the 


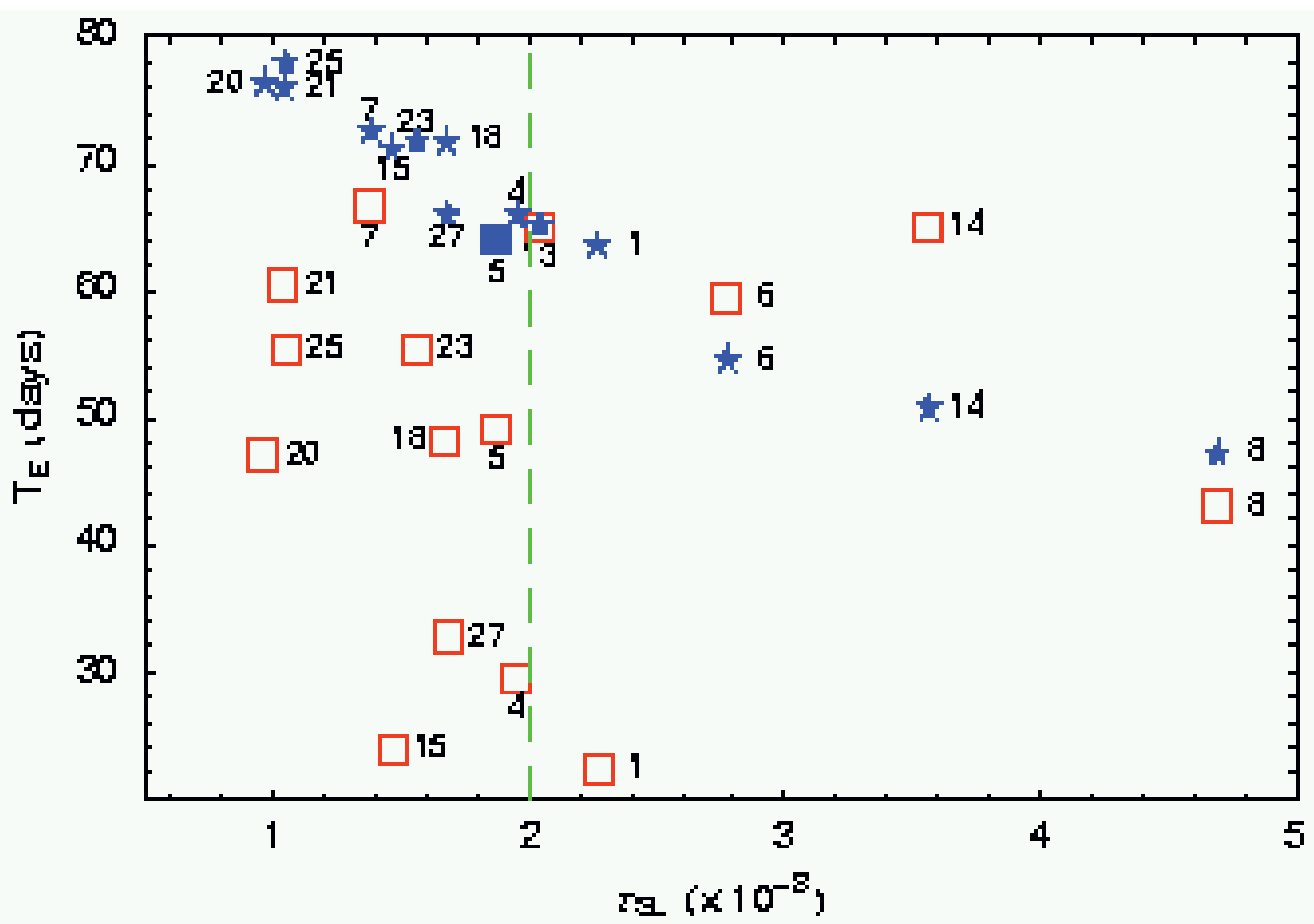

Figure 1. Scatter plot of the observed (empty boxes) values of the Einstein time and of the expected values of the median $T_{\mathrm{E}, 50 \%}$ (filled stars), with respect to the self-lensing optical depth evaluated along the directions of the events. From [22].

measured Einstein times fluctuate around a median value of 59 days, very near to the average value 56 days of the expected medians, ranging from 47 days to 65 days. Let us note, also, the somewhat peculiar position of the event LMC-1, with a very low value of the observed $T_{\mathrm{E}}$; most probably this event is homogeneous to the set at left of the vertical line in Fig. 1 and it has to be included in that cluster. This plot suggests that the cluster of the 9 events including LMC -1 belongs, very probably, to a different population. Clearly, given the large uncertainties and the few events at disposal it is not yet possible to draw sharp conclusions, nevertheless it is plausible that up to 3-4 MACHO events might be due to lenses in LMC, which are most probably low mass stars, but that hardly all events can be due to self-lensing. The most plausible solution is that the events observed so far by the MACHO team are due to lenses belonging to different intervening populations: low mass stars in the LMC, in the thick disk, in the spheroid and some true MACHOs in the halo of the Milky Way and the LMC itself.

Moreover, it emerges that the spatial distribution of the events observed so far shows a near-far asymmetry, which turns out to be compatible with the optical depth calculated for LMC halo objects [22]. The expected characteristics of the lenses belonging to the MACHO population of the Milky Way halo do not match the observed ones. This suggests that this contribution can not explain all the observed candidates as well. Accordingly, we challenged the view that the MACHO dark halo fraction of both the Milky Way and the LMC halos are equal, and indeed we showed that for a MACHO mass in the range 0.1-0.3 $\mathrm{M}_{\odot}$ the LMC halo fraction can be significantly larger than the Milky Way one [26]. In 
this perspective, it might well be that about half of the observed events are due to lenses located in the LMC halo.

\section{Microlensing towards the Galactic bulge region}

To date, the MACHO [27], EROS [28], MOA and OGLE [29] collaborations found several thousands microlensing events towards the Galactic bulge. Considering as sources only the clump giant stars the MACHO team finds a value for the optical depth $\tau=2.17_{-0.38}^{+0.47} \times 10^{-6}$ [30]. Similar values for the optical depth have been found by the OGLE [31] and the EROS [32] teams [33]. The MACHO and EROS collaborations found also several events towards the spiral arm regions [34]. These results are important for studying the structure of our Galaxy and the stellar mass function [35]. Recently, the MOA collaboration [36] found a population of unbound or distant planetary mass population (with masses of a few Jupiter mass) and could better determine the slope of the stellar mass function at low masses.

We addressed the problem of the shape of the initial mass function of the Galactic bulge using the microlensing observations towards the Galactic center by the MACHO, EROS and OGLE collaborations $[35,37]$. In particular, we considered the duration distribution of the microlensing events. Assuming a power law for the initial mass function we studied the slope, as given by the power law index, both in the brown dwarf and in the main sequence ranges. Moreover, we compared the observed and theoretical, based on bulge modelling, optical depth profiles, which turn out to be in quite good agreement [35].

Furthermore, using the current microlensing and dynamical observations of the Galaxy one can set interesting constraints on the dark matter local density and profile slope towards the galactic centre. Assuming state-of-the-art models for the distribution of baryons in the Galaxy, we found, for instance, that the most commonly discussed dark matter profiles (Navarro-Frenk-White and Einasto) are consistent with microlensing and dynamical observations, while extreme adiabatically compressed profiles are robustly ruled out. When a baryonic model that also includes a description of the gas is adopted, our analysis provides a determination of the local dark matter density, $\rho_{0}=0.20-0.56 \mathrm{GeV} / \mathrm{cm}^{3}$ at $1 \sigma$, that is well compatible with estimates given in the literature based on different techniques [38]. Clearly, once more microlensing data will be gathered and thus better galactic models will be constructed the above estimates might become more precise and thus also more interesting.

\section{Microlensing towards globular clusters}

In the last years we analysed the large set of microlensing events detected so far towards the Galactic center with the purpose of investigating whether some of the dark lenses are located in Galactic globular clusters [39]. We found that in some cases the events might indeed be due to lenses located in the globular clusters themselves. We got a rough estimate for the average lens mass of the subset of events being highly aligned with Galactic globular cluster centers and found that, under reasonable assumptions, the deflectors might most probably be either brown dwarfs, M-stars or stellar remnants [40].

In 2000 July/August a microlensing event occurred at a distance of 2.33 arc minutes from the center of the globular cluster M22 (NGC 6656), observed against the dense stellar field of the Milky Way bulge. In order to check the hypothesis that the lens belongs to the globular cluster we made a dedicated observation, using the adaptive optics system NACO at the ESO Very Large Telescope to resolve the two objects - the lens and the source - that participated in the event. The position of the objects measured in July 2011 was in agreement with the observed relative proper motion of M22 with respect 


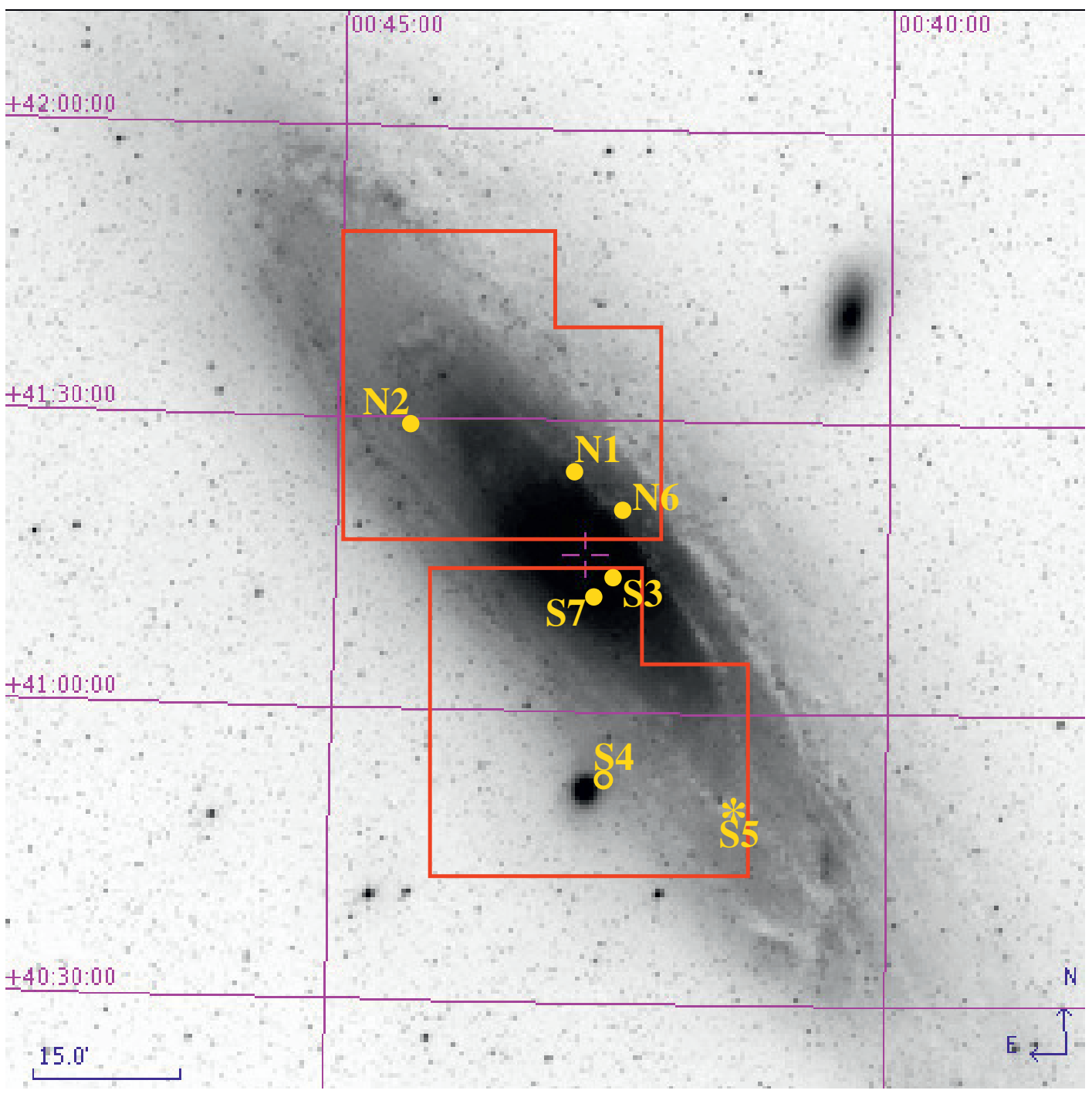

Figure 2. Positions of some microlensing events found so far projected on M31. The solid lines show the contour of the observed fields. Note that the event denoted by S4 almost on the line of sight of M32, a satellite galaxy of M31. From [48].

to the background bulge stars. Based on the brightness of the microlens components we found that the source is a solar-type star located at a distance of $6.0 \pm 1.5 \mathrm{kpc}$ in the bulge, while the lens is a $0.18 \pm 0.01 M_{\odot}$ dwarf member of the globular cluster M22 located at the known distance of $3.2 \pm 0.2$ kpc from the Sun [41]. This is indeed the first confirmed microlens in a globular cluster. It would be desirable to get more observation of globular clusters, indeed few events could already help very much to constrain the low mass star content and thus to get a clear mass budget and information on the stellar mass function of the globular cluster. 


\section{Microlensing events towards M31}

Microlensing searches towards the Andromeda galaxy (M31) have also been proposed [42-44]. In this case one has to use the so-called "pixel-lensing" method, since the source stars are in general no longer resolvable. This makes the subsequent analysis more difficult, however, it allows M31 and other objects, such as M87 [45], to be used as targets [46]. For information on the shape of the dark halo, which is presently unknown, it is important to observe microlensing in different directions as can be done towards M31. Several teams performed searches, in particular the French AGAPE [47], the POINT-AGAPE [48], the MEGA [49], the WeCapp [50, 51] and the PLAN (Pixel Lensing ANdromeda) [52, 53]. All groups found some candidate events consistent with microlensing. As a main result, a high-threshold analysis of the 3 years of POINT-AGAPE data yielded 6 bright, shortduration microlensing events. The observed events are more than expected from self lensing alone, thus leading to the conclusion that at least $20 \%$ of the halo mass in the direction of M31 should be in the form of MACHOs, if their average mass lies in the range 0.5-1 $\mathrm{M}_{\odot}$ [48]. Also the MEGA group presented their results with the detection of 14 candidate events [49]. The observed timescale distribution of the events seems to be consistent with halo lensing dominating in the outer parts of M31.

Within the PLAN collaboration we found during the 2007 season two candidate microlensing events [53]. One event (OAB-N2) was at the end of the observing period, but fortunately the WeCAPP collaboration made observations afterwards. We thus shared the data and the combined analysis shows that with the better coverage the event is nicely confirmed. The improved photometry enabled a detailed analysis in the event parameter space including the effects due to finite source size. The combined results of these analyses allowed us to put a strong lower limit on the lens proper motion. This outcome favours the MACHO lensing hypothesis over self-lensing for this individual event and points the way towards distinguishing between the MACHO and self-lensing hypotheses from larger data sets [54].

The PAndromeda team (a following up of the WeCapp collaboration) using the Pan-STARRS survey done with a 1.8 meter telescope found recently 6 microlensing events towards M31 [55]. The interpretation of these results in terms of limits on the dark matter in form of MACHOs in M31 has, however, not yet been done. More observations are needed to clarify these preliminary results, nonetheless today it is established that the pixel-lensing method works.

\subsection{Pixel-lensing as a way to detect extrasolar planets in M31}

We studied also the possibility to detect extrasolar planets in M31 through pixel-lensing observations, similar to what is done by classical microlensing techniques towards the galactic center [56]. Using a Monte Carlo approach, we selected the physical parameters of the binary lens system, a star hosting a planet, and we calculated the pixel-lensing light curve taking into account the finite source effects. Indeed, their inclusion is crucial since the sources in M31 microlensing events are mainly giant stars. Light curves with detectable planetary features are selected by looking for significant deviations from the corresponding Paczyński shapes. We found that the time scale of planetary deviations in light curves increase (up to 3-4 days) as the source size increases. This means that only few exposures per day, depending also on the required accuracy, may be sufficient to reveal in the light curve a planetary companion. Although the mean planet mass for the selected events is about $2 M_{\text {Jupiter }}$, even small mass planets $\left(M_{\mathrm{P}}<20 M_{\oplus}\right)$ can cause significant deviations, at least in the observations with large telescopes. However, even in the former case, the probability to find detectable planetary features in pixel-lensing light curves is at most a few percent of the detectable events, and therefore many events have to be collected in order to detect an extrasolar planet in M31. Our analysis also supports the 
claim that the anomaly found in the candidate event PA-99-N2 towards M31 [57] can be explained by a companion planetary object of about 6 Jupiter masses orbiting the lens star [56].

\section{Conclusions}

Microlensing has proven in these years to be a very powerful method in various areas of astrophysics: from the determination of the dark matter content in form of MACHOs in the halo of our Galaxy and in the one of M31, for the study of the structure of our Galaxy, the determination of the stellar mass function, particularly in its low mass end, to the detection of planets in the Galaxy and even in the nearby M31. Progress has also been done in the study of stellar atmospheres or the low mass star content of globular clusters. All these topics and maybe others will greatly benefit from more observations and data which will surely come in the next years. Discussion are also underway to use satellites like Euclid for performing microlensing observations towards the Galactic center with the aim to find planets. Microlensing is now a mature technique with many applications which will help solving current astrophysical problems.
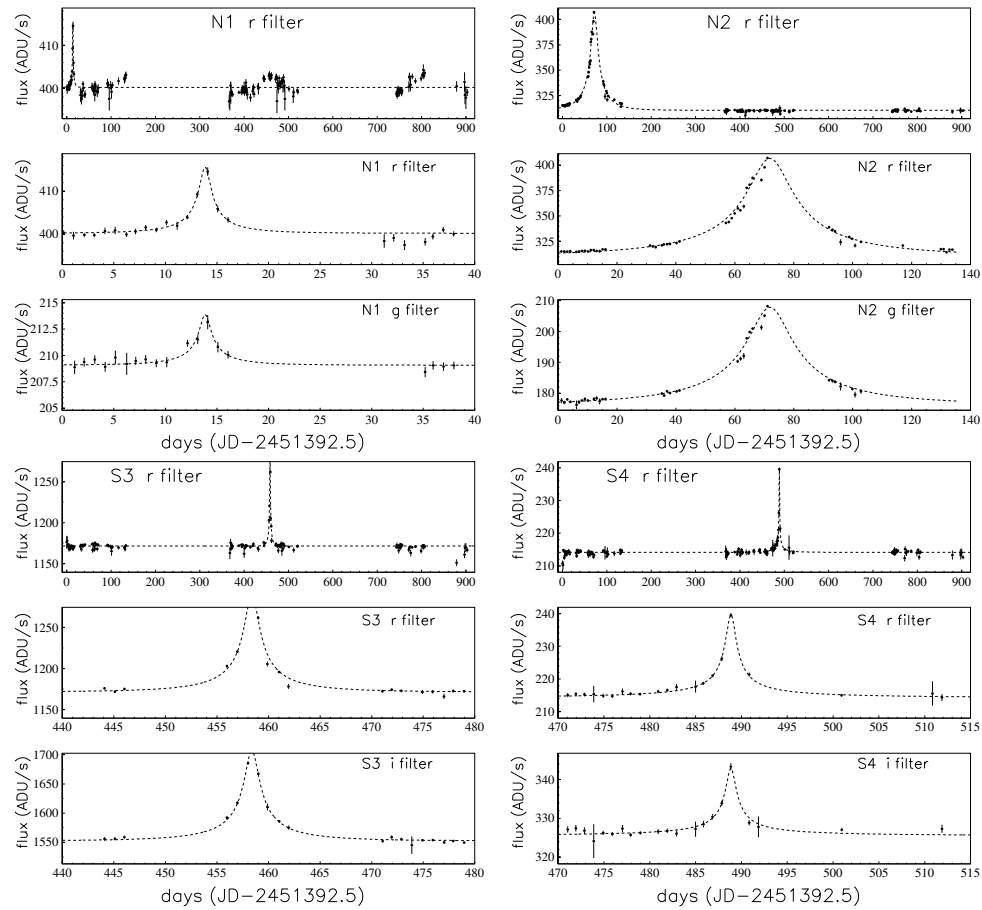

Figure 3. Three years data light curves for 4 microlensing events found by the POINT-AGAPE collaboration. From [48]. 
EPJ Web of Conferences

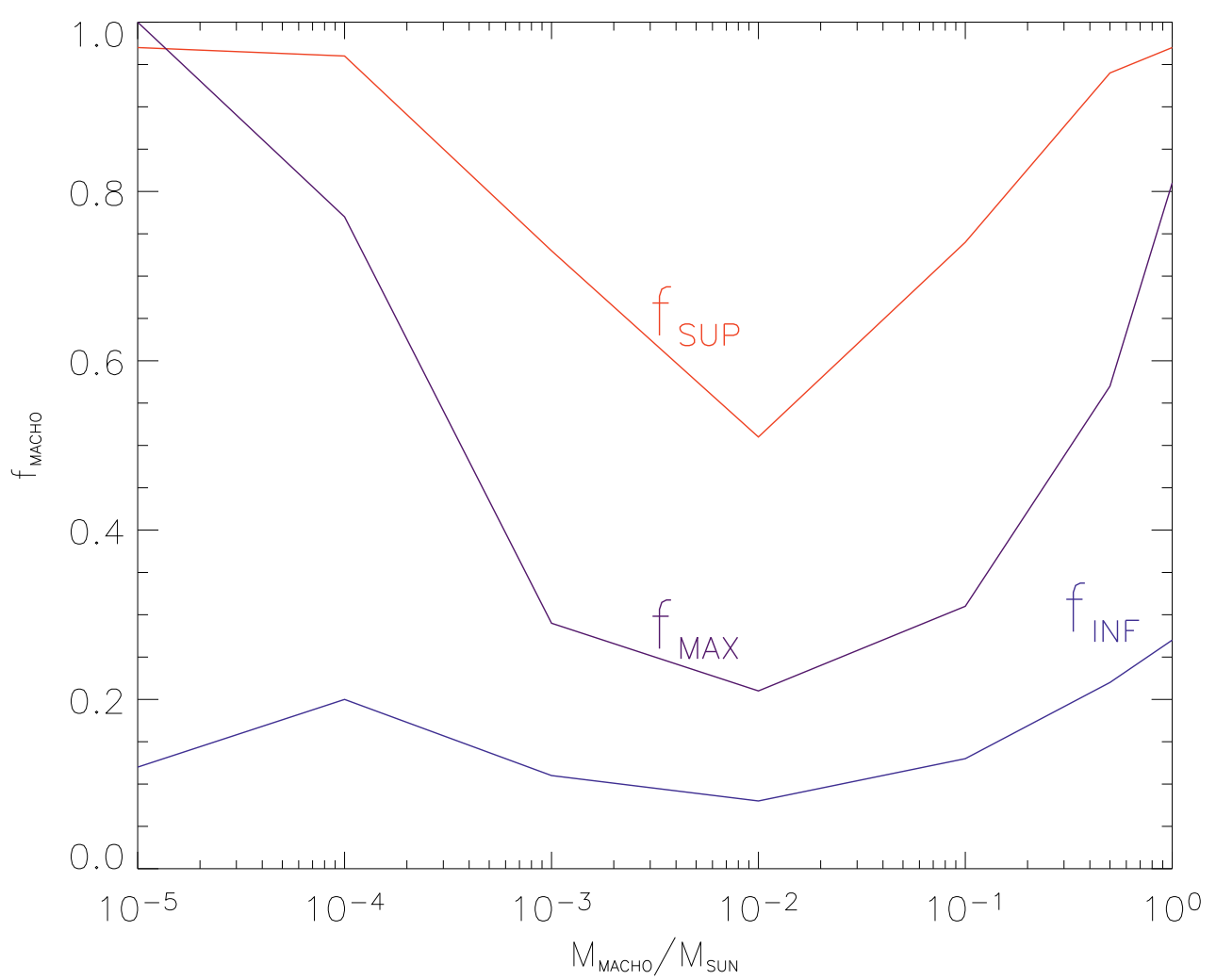

Figure 4. Most probable value, upper and lower 95\% CL limit for the halo fraction as a function of the MACHO mass in solar mass units, based on the POINT-AGAPE results. From [48].

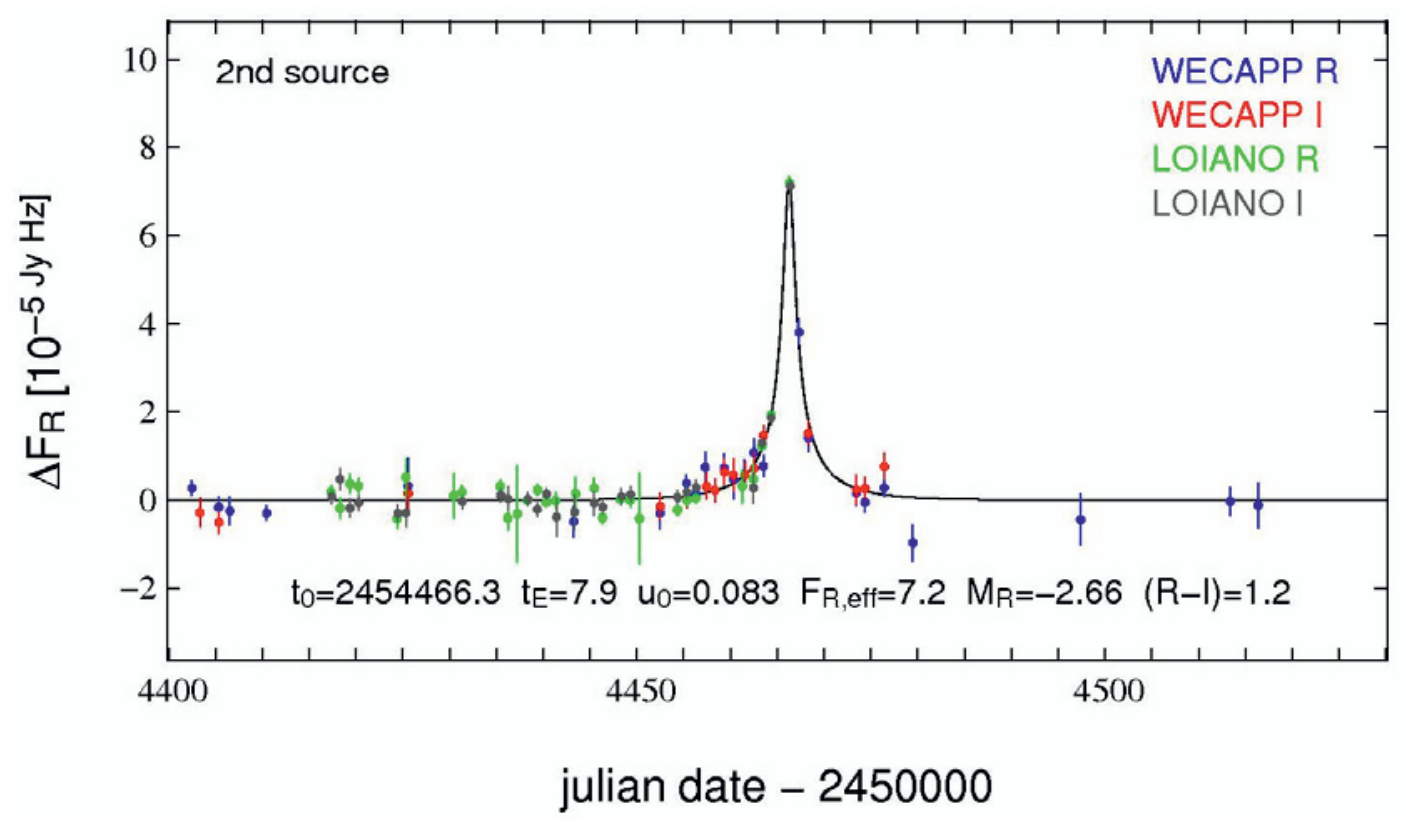

Figure 5. OAB-N2 light curve as obtained by combining PLAN and WeCAPP data. From [54]. 


\section{References}

[1] B. Paczyński, Astrophys. J 304 (1986) 1

[2] A. De Rújula, Ph. Jetzer and E. Massó, MNRAS 250 (1991) 348

[3] A. De Rújula, Ph. Jetzer and E. Massó, Astron. \& Astrophys. 254 (1992) 99

[4] B. Paczyński, Astrophys. J. 371 (1991) L63

[5] K. Griest et al., Astrophys. J. 372 (1991) L79

[6] E. Aubourg et al., Nature 365 (1993) 623

[7] C. Alcock et al., Nature 365 (1993) 621; Astrophys. J. 445 (1995) 133

[8] A. Udalski et al., Acta Astron. 43 (1993) 289

[9] M. Dominik, Gen. Relativ. Gravit. 42 (2010) 2075

[10] G. Ingrosso et al., arXiv 1208.0174 (2012)

[11] Special issue on gravitational lensing, in Gen. Rel. and. Grav. 42, 2010, edited by Ph. Jetzer, Y. Mellier and V. Perlick

[12] D. Bennett, Astrophys. J. 633 (2005) 906

[13] P. Tisserand et al., Astron. \& Astrophys.469 (2007) 387

[14] L. Wyrzykowski et al., MNRAS 413 (2011) 493

[15] S. Calchi Novati and L. Mancini, MNRAS 416 (2011) 1292

[16] L. Wyrzykowski et al., MNRAS 407 (2010) 189

[17] K.C. Sahu, PASP 106 (1994) 942

[18] E. Aubourg, N. Palanque-Delabrouille, P. Salati et al., Astron. \& Astrophys. 347 (1999) 850

[19] N.W. Evans, G.Gyuk, M.S. Turner and J.J. Binney, Astrophys. J 501 (1999) L45

[20] H.S. Zhao and N.W. Evans, Astrophys. J 545 (2000) L35

[21] Ph. Jetzer, L. Mancini and G. Scarpetta, G., Astron. \& Astrophys. 393 (2002) 129

[22] L. Mancini, S. Calchi Novati, Ph. Jetzer and G. Scarpetta, Astron. \& Astrophys. 427 (2004) 61

[23] C. Alcock, R.A. Allsman, D.R. Alves et al., Astrophys. J 541 (2000) 270

[24] C. Alcock, R.A. Allsman, D.R. Alves et al., Astrophys. J 552 (2001) 259

[25] C. Alcock, R.A. Allsman, D.R. Alves et al., Astrophys. J 552 (2001) 582

[26] S. Calchi Novati et al., Astron. \& Astrophys. 459 (2006) 407.

[27] C. Alcock et al., Astrophys. J. 479 (1997) 119

[28] C. Afonso et al., Astron. \& Astrophys. 401 (2003) 145

[29] P.R. Wozniak et al., Acta Astron. 52 (2002) 129

[30] P. Popowski et al., Astrophys. J. 631 (2005) 879

[31] T. Sumi et al., Astrophys. J. 636 (2006) 240

[32] C. Hadamache et al., Astron. \& Astrophys. 454 (2006) 185

[33] M. Moniez, Gen. Relativ. Gravit. 42 (2010) 2047

[34] F. Derue et al., Astron. \& Astrophys. 373 (2001) 126

[35] S. Calchi Novati, F. De Luca, Ph. Jetzer, L. Mancini and G. Scarpetta, Astron. \& Astrophys. 480 (2008) 723.

[36] T. Sumi et al., Nature 473 (2011) 473

[37] L. Grenacher et al., Astron. \& Astrophys. 351 (1999) 775

[38] F. Iocco, M. Pato, G. Bertone and Ph. Jetzer, JCAP 1111 (2011) 029

[39] Ph. Jetzer, M. Straessle and U. Wandeler, Astron. \& Astrophys. 336 (1998) 411

[40] F. De Luca and Ph. Jetzer, Int. J. Mod. Phys. D17 (2008) 2305 
[41] P. Pietrukowicz, D. Minniti, Ph. Jetzer, J. Alonso-Garcia and A. Udalski, Ap. J. Lett. 744 (2012) L18

[42] A.P. Crotts, Astrophys. J. 399 (1992) L43

[43] P. Baillon, A. Bouquet, Y. Giraud-Héraud and J. Kaplan, Astron. \& Astrophys. 277 (1933) 1

[44] Ph. Jetzer, Astron. \& Astrophys. 286 (1994) 426

[45] E. Baltz et al, Astrophys. J. 610 (2004) 691

[46] S. Calchi Novati, Gen. Relativ. Gravit. 42 (2010) 2101

[47] R. Ansari et al., Astron. \& Astrophys. 344 (1999) L49

[48] S. Calchi Novati et al., Astron. \& Astrophys. 443 (2005) 991

[49] J.T.A. de Jong et al., Astron. \& Astrophys. 446 (2006) 885

[50] A. Riffeser et al., Astrophys. J. 599 (2003) L17

[51] A. Riffeser, S. Seitz and R. Bender, Astrophys. J. 684 (2008) 1093

[52] S. Calchi Novati et al., Astron. \& Astrophys. 469 (2007) 115

[53] S. Calchi Novati et al., Astrophys. J 695 (2009) 442

[54] S. Calchi Novati et al., Astrophys. J 717 (2010) 987

[55] C.H. Lee et al., Astron. J 143 (2012) 89

[56] G. Ingrosso et al., MNRAS 399 (2009) 219

[57] J. An et al., Astrophys. J 601 (2004) 845 